\title{
OBSTRUÇÃO URETRAL EM GATOS
}

Keytyanne de Oliveira Sampaio ${ }^{1}$ Grazielle Anahy de Sousa Aleixo Reginaldo Pereira de Sousa-Filho Ellen Cordeiro Bento da Silva ${ }^{1}$

\section{RESUMO}

A obstrução uretral é um quadro clínico emergencial, comumente observado em felinos, que pode resultar em sério comprometimento à sua saúde, como lesões renais agudas. Essa afecção pode ter causas mecânica, anatômica ou funcional, e apresentar como principais sintomas a estrangúria, periúria e lambedura excessiva da genitália. $\mathrm{O}$ diagnóstico é realizado com base no histórico, exame físico e de imagem, como ultrassonografia e radiografia, sendo a identificação da etiologia de suma importância para instituir o tratamento e diminuir os quadros de recidiva. Ao diagnosticar a doença, o início do tratamento deve ser imediato e intensivo, em virtude do risco de evoluir rapidamente para o óbito. Neste sentido, busca-se corrigir os distúrbios hidroeletrolíticos, fornecer analgesia e realizar a desobstrução uretral, havendo uma variação neste último procedimento, de modo que, a escolha do método mais indicado pode variar de acordo com cada caso. Com base na complexidade em seu estabelecimento, diagnóstico e tratamento, foi objetivado com esse artigo de revisão reunir informações atuais e relevantes, relacionadas ao quadro de obstrução uretral em gatos, procurando enfatizar aspectos clínico, cirúrgico e etiológico da doença, assim como, orientar médicos veterinários no tratamento e prevenção da mesma.

Palavras-chave: cateterização, gato doméstico, uretra.

\section{URETHRAL OBSTRUCTION IN CATS}

\begin{abstract}
Urethral obstruction is an emergency clinical condition, commonly observed in cats, that can result in serious commitment of their health, such as acute kidney damage. This condition can have mechanical, anatomical or functional causes, presenting as main symptoms stanguria, periuria and excessive licking of genitals. The diagnosis is based on the history, physical and image exams, such as ultrasound and radiography, being the identification of etiology of great importance to institute the treatment and reduce recurrence. After diagnosing the disease, initiation of treatment needs to be immediate and intensive, because of the risk of evolving quickly to death. In this regard, it's necessary to correct hydroelectric disturbances, provide analgesia and perform a urethral clearance, however there is a variation in this last procedure, so the choice of the most suitable method may vary according to each case. Based on the complexity of its establishment, diagnosis and treatment, this review aimed to gather current and relevant information related to urethral obstruction in cats, intending to emphasize clinical and etiological symptoms of the disease, as well as to guide veterinarians in its treatment and prevention.
\end{abstract}

Keywords: catheterization, domestic cat, urethra.

\footnotetext{
${ }^{1}$ Universidade Federal Rural de Pernambuco. Keyttyanne_sampaio@ hotmail.om
} 


\section{OBSTRUCCIÓN URETRAL EN GATOS}

\section{RESUMEN}

La obstrucción uretral es una condición clínica de emergencia, comúnmente vista en gatos, que puede resultar en un deterioro grave de su salud, como daño renal agudo. Esta condición puede tener causas mecánicas, anatómicas o funcionales, y presentar extrañeza, periuria y lamido excesivo de los genitales como síntomas principales. El diagnóstico se realiza con base en la historia, examen físico y de imagen, como ecografía y radiografía, y la identificación de la etiología es de suma importancia para instituir el tratamiento y reducir la recurrencia. Al diagnosticar la enfermedad, el inicio del tratamiento debe ser inmediato e intensivo, debido al riesgo de evolucionar rápidamente a la muerte. En este sentido, buscamos corregir las alteraciones hidroelectrolíticas, aportar analgesia y realizar el aclaramiento uretral, con una variación en este último procedimiento, por lo que la elección del método más adecuado puede variar según cada caso. A partir de la complejidad de su establecimiento, diagnóstico y tratamiento, este artículo de revisión tuvo como objetivo recopilar información actual y relevante relacionada con la condición de obstrucción uretral en gatos, buscando enfatizar los aspectos clínicos, quirúrgicos y etiológicos de la enfermedad, así como orientar a los médicos veterinarios en su tratamiento y prevención.

Palabras clave: cateterismo, gato doméstico, uretra.

19

\section{INTRODUÇÃO}

A obstrução uretral é uma emergência médica diagnosticada entre 10 a $22 \%$ dos gatos com doença do trato urinário inferior (1), podendo causar sérios prejuízos em termos reprodutivos e sanitários, com manifestações clínicas como disúria, estrangúria, hematúria, dor e anorexia (2).

A origem da obstrução uretral pode ser de cunho mecânico, anatômico ou funcional. Dentre as causas mecânicas, a oclusão da uretra por urólitos e pela formação de tampões são os motivos mais frequentes. Por outro lado, os defeitos na formação da uretra ou pênis e o intenso processo inflamatório, constituem, respectivamente, os fatores anatômicos e funcionais mais comuns (3).

Cada vez mais trabalhos procuram informações sobre a patogênese e os fatores predisponentes das obstruções uretrais. Nevins et al. (4) e Borges et al. (5) relacionam tal desordem com a castração precoce dos felinos, embora ainda não esteja bem estabelecido o mecanismo que justifique essa suspeita. Em virtude disso, surge o temor aos possíveis efeitos adversos associados à interrupção hormonal prematura, fato que faz com que no Brasil seja preconizado a castração de gatos apenas após a puberdade (6).

Diante da alta incidência, complexidade e importância da doença obstrutiva felina, objetivou-se com esse artigo de revisão, reunir trabalhos atuais e relevantes relacionados ao quadro de obstrução uretral nessa espécie, procurando compilar as informações e divulgar os principais aspectos clínicos e etiológicos da enfermidade, para desta maneira, orientar médicos veterinários quanto aos tratamentos, bem como sobre as formas de prevenção.

\section{DESENVOLVIMENTO}

\section{Considerações gerais}

O termo doença do trato urinário inferior dos felinos (DTUIF) é utilizado para descrever distúrbios na bexiga e uretra, sendo considerada uma das afecções mais comuns na 
clínica desses animais (2). Esta possui etiologia multifatorial e muitas vezes indeterminada, com manifestação de sintomatologias semelhantes, relacionadas ao trato urinário inferior (7).

A DTUIF pode ser classificada em obstrutiva e não obstrutiva (3) sendo a forma obstrutiva diagnosticada em 17 a $58 \%$ dos gatos com a enfermidade $(3,8)$.

As principais causas não-obstrutivas são a idiopática, urolitíase e infecção bacteriana, as quais, comumente, são autolimitantes e, portanto, marcadas por resolução espontânea (9). A obstrutiva se manifesta com a interrupção do fluxo, em virtude da presença de tampões ou urólitos na luz da uretra, defeitos anatômicos da mesma, bem como edemas, fibroses, traumas ou infecções induzidas pela passagem de cateter vesical $(3,10)$. Como consequência, há a cessação da filtração renal, tornando-se, portanto, uma afecção urológica emergencial e potencialmente fatal (3).

\section{Fisiopatologia e epidemiologia}

A obstrução uretral é frequentemente observada em gatos machos, devido a conformação anatômica da uretra, a qual é mais longa e estreita, quando comparada às fêmeas $(2,5)$. Alguns trabalhos relatam uma possível relação entre este quadro e a castração pediátrica, embora ainda não esteja bem estabelecido o mecanismo que justifique tal associação (4).

Diante da problemática acima descrita, tem se procurado desvendar os reais fatores determinantes para o surgimento de obstruções uretrais na espécie. Lee e Drobatz (11), em estudo retrospectivo com 223 gatos com quadro de obstrução urinária, constataram que 90\% eram castrados. Já em pesquisa realizada por Porters et al. (12), estes demostraram que a incidência do quadro é semelhante entre gatos submetidos à castração pré-púberes e póspúberes. Em outro trabalho realizado por Herron et al. (13), ao avaliarem o efeito da castração na uretra masculina e tecido periuretral, eles concluíram que os gatos intactos apresentavam epitélio uretral mais espesso do que os castrados. Por outro lado, Borges et al. (5) não verificaram diferenças para o diâmetro luminal e espessura do epitélio uretral de machos castrados e não castrados. Apesar disso, segundo eles, a densidade das fibras elásticas no corpo esponjoso, na túnica albugínea e no corpo cavernoso de animais submetidos à orquiectomia foi menor do que nos intactos. Em contrapartida, houve aumento na densidade das fibras colágeno no corpo esponjoso de animais castrados, sugerindo ser esse um fator predisponente para reduzir a complacência da região periuretral e predispor à doença obstrutiva.

De acordo com Ferreira et al. (14), a castração pode levar à obesidade e consequente, diminuição da atividade física, o que parece predispor à DTUIF. Isso se deve ao fato de que a remoção das gônadas diminui o seu metabolismo (pelo seu papel endócrino), de modo que a hipoatividade promove a diminuição da frequência de micção, favorecendo a formação de cristais e, posteriormente, cálculos urinários (15).

Acredita-se ainda que o estresse seja um ponto chave à indução de mudanças no consumo de alimento e ingestão de água na espécie, pois fatores estressantes são relacionados à prevalência de quadros de cistitite idiopática e de formação de urólitos (16).

A obstrução uretral por tampões decorre da formação dessas estruturas a partir de infecção urinária ou inflamação na bexiga com cristalúria, o que resulta na agregação de proteínas, cristais, leucócitos e glóbulos vermelhos. Outra teoria sugere que a inflamação crônica leva à uma diminuição da integridade vascular da bexiga urinária, com aumento da concentração de proteínas na urina e de seu pH, com consequente formação dos tampões (17). Le Boedec et al. (18) relataram sobre a cistite pseudomembranosa, grave processo inflamatório que ocasiona o descolamento de mucosa vesical, levando à obstrução uretral.

Apesar da urina na bexiga e na região proximal da uretra ser, geralmente, 
1 considerada estéril, tem-se observado infecções do trato urinário (ITU) causadas por 2 contaminação retrógrada das bactérias presentes nas fezes (19). Portanto, a cistite bacteriana 3 deve ser sempre considerada e investigada nas doenças obstrutivas da uretra (20,21), estando 4 presente em média em $2 \%$ a $12 \%$ dos casos $(3,22)$. Outros estudos, por sua vez, mostram que 5 a percentagem de gatos com infecção urinária podem ser bem maiores e chegar a $45 \%$ dos gatos com sintomas de DTUIF $(3,8,23)$.

\section{Sinais clínicos}

Os sinais clínicos apresentados pelos pacientes acometidos pela obstrução uretral variam de acordo com a duração e grau da obstrução (24). As principais manifestações clínicas apresentadas são diminuição na ingestão de água, polaciúria, estrangúria, periúria, lambedura excessiva da genitália, desidratação, fraqueza, depressão e pênis hiperêmico, edemaciado e exposto (14).

Em casos mais graves, onde há uma obstrução total e a mesma permanece por mais de 36 a 48 horas, sinais de azotemia pós-renal, como vômito, diarreia e anorexia, podem ser observados $(10,25)$. Em pacientes mais debilitados, pode ser constatado pulso fraco e filiforme, hipotermia, mucosas pálidas e ressecadas (26). Na ausculta cardíaca, é possível verificar bradicardia, decorrentes de alterações eletrolíticas, como hipercalemia, contudo, mesmo na ausência da bradicardia, os valores de potássio sérico podem estar aumentados (27).

Adicionalmente, na avaliação física, por meio de palpação abdominal na região caudal, pode-se revelar uma bexiga urinária firme e desconforto por parte do animal obstruído (9).

\section{Diagnóstico}

O diagnóstico de DTUIF obstrutiva deve ser baseado nos sinais clínicos, histórico, e exames físico $(17,20)$, sendo também a ultrassonografia uma importante ferramenta na identificação de alterações causadoras ou consequentes do processo. Nesse exame de imagem podem ser observados o espessamento da parede da bexiga urinária, sedimentos urinários, hidroureter, hidronefrose, coágulos sanguíneos luminais e urólitos (4).

Assim como a ultrassonografia, as radiografias abdominais simples são, frequentemente, utilizadas para o diagnóstico de obstrução uretrais por cálculos. No entanto, para urólitos com menos de três milímetros de diâmetro ou radioluscentes, isso só é possível através da técnica radiográfica contrastada, que permite ainda a identificação de massas, persistência do úraco, coágulos sanguíneos e estenoses (28).

Muitos são os exames complementares, necessários para avaliar o paciente com obstrução uretral. Dentre ele, merece destaque a análise hematológica, através da qual pode ser identificada a elevação dos níveis do hematócrito e da proteína total plasmática, diante do quadro de desidratação (26).

As dosagens de ureia e creatinina séricas são indispensáveis à avaliação da função renal de animais com obstrução uretral, devendo ser executada conjuntamente com a determinação da glicose, cálcio, fósforo, sódio e potássio. Isso possibilita determinar o quadro clínico do paciente, especialmente quando associado à gasometria venosa (9). Lee e Drobatz (11) relataram que a maioria dos gatos com obstrução uretral apresentam alterações leves nos eletrólitos e gases sanguíneos, estando a hipercalemia grave ( $>8,0 \mathrm{mmol} / \mathrm{L})$ presente em $12 \%$ dos animais afetados, geralmente em conjunto com acidemia e baixas concentrações de cálcio ionizado.

Em pacientes com obstrução uretral há aumento da pressão vesical, ocasionando 
elevação da pressão na cápsula de Bowman e diminuição da taxa de filtração glomerular. Consequentemente, há aumento nos níveis séricos de ureia e creatinina (azotemia), além de hipercalemia progressiva. A partir daí é gerada insuficiência renal aguda, de origem pós-renal, que é potencialmente reversível, caso seja realizada a desobstrução precoce das vias urinárias $(29,30)$.

Os valores elevados de creatinina e ureia, observados nos casos de doenças uretrais obstrutivas, são, geralmente, associados à crise urêmica $(3,14)$. Tal disfunção renal, pode levar à acidose metabólica, pelo acúmulo de íons de hidrogénio $\mathrm{H}+$, e diminuição da concentração de íons de sódio $(\mathrm{Na}+)$, necessário para a troca com os íons de potássio $(\mathrm{K}+)$, o que gera o quadro de hipercalemia (31).

Os distúrbios hidroeletrolíticos, metabólicos e acido-básicos resultam em graves complicações para o paciente com DTUIF, incluindo o óbito (32), e assim, a determinação de tais variáveis é um indicativo do estado de saúde do animal. Em estudo realizado por Neri et al. (33), foi observado que pacientes obstruídos a mais de 36 horas apresentavam valores maiores de creatinina, fósforo, magnésio, potássio e lactato, quando comparado com animais obstruídos há menor tempo.

Com relação à urinálise, frequentemente são observados proteinúria, glicosúria, hematúria, leucocitúria, piúria e cristalúria, decorrentes do quadro de inflamação, infeção, necrose ou trauma no trato urinário $(7,33)$. Adicionalmente é possível identificar uma relação proteína-creatinina urinárias aumentada, achados que sugerem um agravamento da lesão renal, conforme o tempo de obstrução (7). Em estudo realizado por Brabson et al. (5), foi evidenciada uma relação da cor da urina com o grau de danos na bexiga urinária, de modo que quanto mais escura a urina, maior o tempo da obstrução e grau das lesões metabólicas (34).

Associado à urinálise, é importante realizar uma urocultura, principalmente nos gatos mais velhos, para ser possível identificar o agente infecioso (quando presente) e instituir uma terapêutica adequada (35). Sabe-se, entretanto, que é a Escherichia coli é o patógeno mais comumente encontrado nos casos de obstrução $(22,36)$.

\section{Tratamento}

O tratamento da obstrução uretral deve ser imediato e intensivo, em virtude do risco do paciente evoluir para o óbito. Ele se baseia na correção dos distúrbios hidroeletrolíticos, na desobstrução uretral e na analgesia $(17,37)$. Com a finalidade de reestabelecer os equilíbrios ácido-básicos e eletrolíticos, bem como estimular a diurese, principalmente nos felinos clinicamente comprometidos, deve ser realizada fluidoterapia $(38,39)$.

Dentre as soluções eletrolíticas que contribuem para a correção da acidose metabólica, o ringer com lactato representa a melhor escolha, por permitir o restabelecimento de fluídos em gatos obstruídos e recompor a homeostase renal mais rápido que a solução salina. Além disso, atua no controle da acidose metabólica, diante do efeito alcalinizante do lactato (39).

Em pacientes com acidose metabólica grave, hipercalemia e azotemia pós-renal, é recomendada a infusão de ringer com lactato de $20 \mathrm{~mL} / \mathrm{kg} / \mathrm{hora}$, nas seis primeiras horas. Nas seis a 12 horas seguintes, a taxa de fluidoterapia deve ser reduzida para $15 \mathrm{~mL} / \mathrm{kg} / \mathrm{hora}$, entre 12 a 24 horas para $10 \mathrm{~mL} / \mathrm{kg} /$ hora e $5 \mathrm{~mL} / \mathrm{kg} /$ hora nas últimas 24 a 48 horas (39). Adicionalmente, de acordo com o estado clínico do paciente, pode haver a necessidade de efetuar infusão de sais de cálcio, glicose ou glicose seguida de insulina, para a correção dos distúrbios hidroeletrolíticos (40).

Diante do intenso quadro de dor observado nos animais com obstrução uretral, é crucial a instituição de protocolo analgésico, sendo recomendado que a terapia com antiinflamatórios não esteroidais (AINE's) seja cautelosa, uma vez que pode agravar injúrias 
renais previamente instaladas (40). Além disso, Dorsch et al. (41) afirmaram que o uso de AINE's na dose recomendada para gatos não parecem ter efeito analgésico e antiinflamatório.

O uso de antiespasmódicos de musculatura lisa pode ser indicado para reduzir o risco de recorrência de obstrução uretral. Dentre tais fármacos se destacam a acepromazina, na dose de 0,02 a $0,05 \mathrm{mg} / \mathrm{kg}$, por via intravenosa (IV), intramuscular (IM) ou subcutânea (SC), ou de 1,0 a 3,0 mg/kg por via oral (VO), a cada quatro a seis horas; prazosina, na dose de 0,25 a 1,0 $\mathrm{mg} /$ gato, VO, a cada oito a 12 horas; e o fenoxibenzamin, na dose de 0,5 a $1,0 \mathrm{mg} / \mathrm{kg}$, VO, a cada 12 ou 24 horas, por até cinco dias $(35,42)$. Para a escolha da intervenção correta, a fim de solucionar a obstrução uretral, é imprescindível identificar a localização da obstrução e a determinação da causa da mesma (43). Algumas estratégias terapêuticas possíveis são a massagem peniana, a cateterização uretral, a hidropropulsão e a compressão vesical, na tentativa de deslocar os tampões uretrais e urólitos (44), sendo essas técnicas conservativas 14 (Fig. 01).

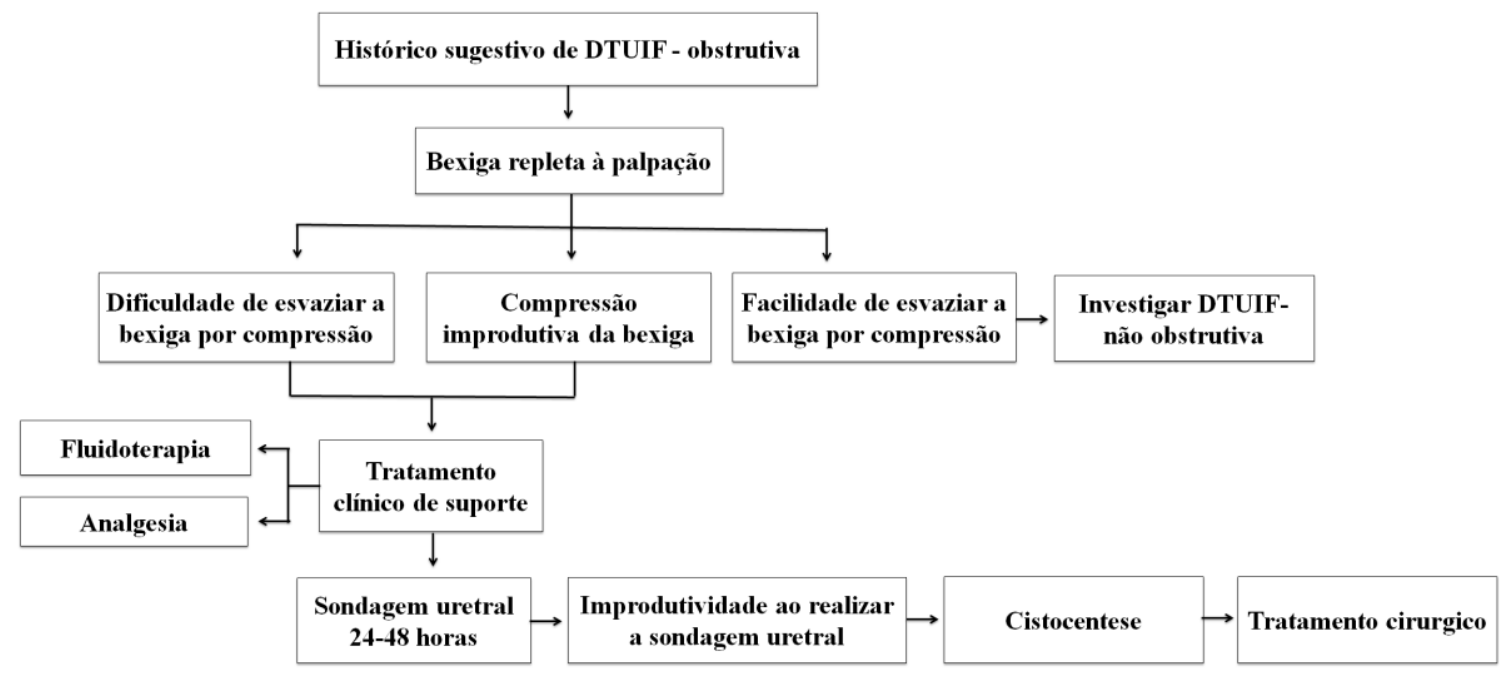

Figura 01: Fluxograma do tratamento de obstrução urinária em felino.

Quando a massagem peniana é ineficiente na desobstrução, opta-se pela cateterização uretral. Esta última deve ser feita de forma asséptica, através da introdução apenas da porção inicial da sonda na uretra peniana, com posterior injeção de solução fisiológica. O objetivo é promover a desobstrução por hidropropulsão, sempre com cuidado para que a introdução forçada do cateter não traumatize o tecido e, assim, ocasione estenose ou ruptura da parede uretral (45).

Após a devida desobstrução, a sonda empregada inicialmente é substituída por outra mais flexível (9), a qual deve permanecer por 24 a 48 horas no local, em sistema fechado, a fim de reduzir a incidência de infecção bacteriana (23). A permanência da sonda é indicada em casos graves de hematúria e uremia, nos procedimentos de difícil desobstrução ou quando, após a desobstrução do fluxo urinário, existe uma grande quantidade de debris e o fluxo de urina normal não foi restabelecido $(9,24)$. Isso se deve ao menor risco de re-obstrução uretral, quando comparado à desobstrução por cateterização única (46).

A cateterização pode não ser efetiva quando na presença de urólitos que não se deslocam e nas estenoses uretrais (47). Nessas situações a cistocentese pode ser indicada, apesar dos relatos de risco de ruptura do órgão, o que decorre da fragilidade de sua parede frente ao processo inflamatório e a tensão causada pela afecção (26).

Nos casos em que a obstrução uretral não responde ao tratamento médico ou o 
paciente apresentar episódios recorrentes, bem como lesões irreversíveis na mucosa da uretra peniana como estenoses, rupturas, traumas e neoplasias, o procedimento cirúrgico através da uretrostomia, é recomendado $(48,49)$. Essa intervenção visa criar um novo óstio uretral e pode ser executada de diferentes formas, com destaque para a técnica perineal, que é indicada quando a obstrução está na uretra peniana, e para a pré-púbica, utilizada quando o processo obstrutivo está na uretra pélvica (50).

As técnicas de uretrostomia podem apresentar complicações como incontinência urinária, infeções urinárias, dermatite peristomal, vazamento de urina subcutânea e estenose (51). Existem relatos de incidência de infecção urinária, a longo prazo em cerca de 17 a $77 \%$ dos pacientes que passaram pelo procedimento cirúrgico $(52,53)$. A quebra na integridade das barreiras anatômicas, através da retirada do esfíncter uretral externo, pode implicar em migração de bactérias para as vias urinárias justificando as infecções (54).

Alguns autores relatam ainda que, após a uretrostomia, alguns animais podem apresentar estenose, sendo indicado reintervenção e colocação de stents uretrais (43). Apesar disso, de acordo com Corgonzinho et al. (2007) (10), a uretrostomia perineal parece ter bons resultados, desde que realizada por cirurgiões experientes e associada ao pós-operatório com o uso de colar elizabetano e sem emprego de sondas uretrais, visto que aumentam o processo inflamatório, contribuem para a maior incidência de estenoses e são uma importante fonte de infecções.

Independente da técnica empregada para a desobstrução uretral, o paciente deve ser monitorado quanto a produção de urina, hidratação e peso corporal, com o objetivo de que se possa individualizar a fluidoterapia de reposição (55). Neste contexto, animais que passaram por quadros obstrutivos devem ser submetidos a um manejo clínico através do aumento na ingestão de água $(56,57)$, o que pode ser obtido pelo fornecimento de dietas com alta concentração de líquido, reduzindo desta forma a concentração urinária e prevenindo a formação de cálculos urinários (58). Em associação, podem ser indicadas dietas terapêuticas que buscam alterar o $\mathrm{pH}$ urinário, reduzir concentração dos cristalóides litogênicos e aumentar a solubilidade dos mesmos (59).

O manejo ambiental de gatos submetidos à uretrostomia é fundamental e marcado pelo aumento do número de caixas sanitárias de areia (número de caixas = número de gatos +1 ) e bebedouros, higienização frequente e melhor disposição, usando locais frescos, calmos e facilmente acessíveis $(58,60)$. Tais cuidados evitam novas manifestações de obstrução uretral, uma vez que é alta a incidência de recidiva (33\%) e mortalidade (entre 5,8\% e 8,9\%) dos animais acometidos $(2,11,37)$.

\section{CONSIDERAÇÕES FINAIS}

Com base nas informações descritas, conclui-se que a obstrução urinária em gatos é uma emergência médica na qual o diagnóstico e tratamento precoce são essenciais para favorecer o prognóstico. Os impactos negativos e a grande incidência na clinica de felinos, torna essa enfermidade de grande importância e com isso, ressalta a necessidade de execução de pesquisas com a intenção de desvendar as causas e consequências da doença.

\section{REFERÊNCIAS}

1 Trangerud C, Ottesen N, Eggertsdo AV. Causes of lower urinary tract 354 disease in Norwegian cats. J Feline Med Surg. 2011;355:410-7.

2 Segev G, Livne H, Ranen E, Lavy E. Urethral obstruction in cats: predisposing factors, clinical, clinicopathological characteristics and prognosis. J Feline Med Surg. 2011;13:101-8. 
13 Saevik BK, Trangerud C, Ottesen N, Sorum H, Eggertsdottir AV. Causes of lower urinary 2 tract disease in Norwegian cats. J Feline Med Surg. 2011;13:410-7.

34 Nevins JR, Mai W, Thomas E. Associations between ultrasound and clinical findings in 87 4 cats with urethral obstruction. Vet Radiol Ultrasound. 2015;56:439-47.

55 Borges NCS, Sampaio MAP, Pereira VA, Figueiredo MA, Chagas MA. Effects of 6 castration on penile extracellular matrix morphology in domestic cats. J Feline Med Surg. $7 \quad 2017 ; 215: 1-6$.

86 Silva TC, Barretot BM, Andradem B, Miranda ALS, Guimarães-Bassolia CD. 9 Conhecimento e percepção dos médicos veterinários do hospital veterinário da UFRPE sobre 10 a castração pediátrica. Rev Educ Contin Med Vet Zootec. 2016;13:72.

7 Defauw PA, Maele IV, Duchateau L. Risk factors and clinical presentation of cats with feline idiopathic cystitis. J Feline Med Surg. 2011;13:967-75. Evaluation of clinical signs and causes of lower: urinary tract disease in Polish cats. Vet Med (Praha). 2017;62:386-93.

169 Costa FVA. Contribuição ao estudo da doença do trato urinário inferior feline. MEDVEP 17 Rev Cientif Med Vet Pequenos Anim Anim Estimação. 2009;7:448-63.

1810 Corgozinho KB, Souza HJM, Pereira AN, Belchior C, Silva MA, Martins MCL, et al. 19 Catheter-introduced urethral trauma in cats with urethral obstruction. J Feline Med Surg. $20 \quad 2007 ; 9: 481-6$.

11 Lee JA, Drobatz KJ. Characterization of the clinical characteristics, electrolytes, acid-base, and renal parameters in male cats with urethral obstruction. J Vet Emerg Crit Care (San Antonio). 2003; 13:227-33.

12 Porters N, Polis I, Moons CPH, Maele IV, Ducatelle R, Goethals K, et al. Relationship between age at gonadectomy and health problems in kittens adopted from shelters. Vet Rec Open. 2015;176:572-6.

13 Herron MA. The effect of prepubertal castration on the penile urethra of the cat. J Am Vet Med Assoc. 1972;160:208-11.

14 Ferreira GS, Carvalho MB, Avante MB. Características epidemiológicas, clínicas e laboratoriais de gatos com sinais de doença do trato urinário inferior. Farriers Mag. 2014;19:42-50.

15 Larsen JA. Risk of obesity in the neutered cat. J Feline Med Surg. 2017;19:779-83.

16 Lund HS, Eggertsdóttir AV. Recurrent episodes of feline lower urinary tract disease with different causes: possible clinical implications. J Feline Med Surg. 2019;21:590-4.

17 Hostutler RA, Chew DJ, Dibatola SP. Recent concepts in feline lower urinary tract disease. Vet Clin North Am Small Anim Pract. 2005;35:147-70.

18 Le Boedec K, Pastor ML, Lavoue R. Pseudomembranous cystitis, an unusual condition associated with feline urine outflow obstruction: four cases. J Feline Med Surg. 201;13:58893.

19 Siddiqui H, Nederbragt AJ, Lagesen K, Jeansson SL, Jakobsen KS. Assessing diversity of 
120 Eggertsdóttir AV, Saevik BK, Halvorsen I, Sorum H. Occurrence of occult bacteriuria in 2 healthy cats. J Feline Med Surg. 2011;13:800-3.

321 Puchot ML, Cook AK, Pohlit C. Subclinical bacteriuria in cats: prevalence, findings on 4 contemporaneous urinalyses and clinical risk factors. J Feline Med Surg. 2017;19:1238-44. 22 Eggertsdottir AV, Lund HS, Krontveit R, Sorum H. Bacteriuria in cats with feline lower urinary tract disease: a clinical study of 134 cases in Norway. J Feline Med Surg. 2007;9:45865 .

23 Dorsch R, Remer C, Sauter-Louis C, Hartmann K. Feline lower urinary tract disease in a German cat population. A retrospective analysis of demographic data causes and clinical signs. Tierarztl Prax Ausg G Grosstiere Nutztiere. 2014;42:231-9.

24 Galvão ALB, Ondani AC, Frazílio FO, Guadalupe SF. Obstrução uretral em gatos machos - revisão literária. Acta Vet Bras. 2010;4:1-6.

25 Dowers K. Nonobstructive idiopathic feline lower urinary tract disease: how to approach a puzzling disorder. Vet Med [Internet]. 2009 [cited 2020 Sept 2]. Available from: https://www.dvm360.com

26 Walker D. Feline urethral obstruction: a clinical refresh. Ir Vet J. 2009;62:198-202.

27 Tag TL, Thomas KD. Electrocardiographic assessment of hyperkalemia in dogs and cats. J Vet Emerg Crit Care (San Antonio). 2008;18:61-7.

28 Hecht S. Diagnostic imaging of lower urinary tract disease. Vet 20 Clin North Am Small Anim Pract. 2015;45:639-63.

29 Kellum JA. Acute kidney injury. Crit Care Med. 2008;36:141-52.

30 Fischer JR, Lane IF, Stokes J. Acute postrenal azotemia: etiology, clinicopathology, and pathophysiology. Compend Contin Educ Vet. 2009;31:520-30.

31 Vieira Neto OM, Moysés Neto M. Distúrbios do equilíbrio hidroeletrolítico. Medicina (Ribeirão Preto). 2003;36:325-37.

32 Reche-Jr A, Hagiwara MK. Semelhanças entre a doença idiopática do trato urinário inferior dos felinos e a cistite intersticial humana. Cienc Rural. 2004;34:315-21.

33 Neri AM, Machado LHA, Okamoto PTG, Filippi MG, Takahira RK, Melchert A, et al. Routine screening examinations in attendance of cats with obstructive lower urinary tract disease. Top Companion Anim Med. 2016;31:140-5.

34 Brabson TL, Bloch CP, Johnson JA. Correlation of gross urine color with diagnostic findings in male cats with naturally occurring urethral obstruction. J Feline Med Surg. 2015; $17: 453-7$.

35 Gunn-Moore DA. Feline lower urinary tract disease. J Feline Med Surg. 2003;5:133-8.

36 Bailiff NL, Nelson RW, Feldman EC, Westropp JL, Ling GV, Jang SS. Frequency and risk factors for urinary tract infection in cats with diabetes mellitus. J Vet Intern Med. 2006;20:850-5.

3837 Gerber B, Eichenberger S, Reusch CE. Guarded log-term prognosis in male cats with 39 urethral obstruction. J Feline Med Surg. 2008;10:16-23.

4038 Norman BC, Côté E, Barrett KA. Wide-complex tachycardia associated with severe 41 hiperkalemya in three cats. J Feline Med Surg. 2006;8:372-8. 
39 Cunha MG, Freitas GC, Carregaro AB, Gomes K, Cunha JP, Beckmann DV, et al. Renal and cardio-respiratory effects of treatment with lactated Ringer's solution or physiologic saline $(0.9 \% \mathrm{NaCl})$ solution in cats with experimentally induced urethral obstruction. Am J Vet Res. 2010;71:840-6.

40 Rieser TM. Urinary tract emergencies. Vet Clin North Am Small Anim Pract.

6 2005;35:359-73.

41 Dorsch R, Zellner F, Schulz B, Sauter-Louis C, Hartmann K. Evaluation of meloxicam for the treatment of obstructive feline idiopathic cystitis. J Feline Med Surg. 2016;18:925-33.

42 Westropp JL. Cats with lower urinary tract sings. Vet Focus. 2007;17:10-7.

43 Drebing MR, Fui Y, Mayank S, Ruth D, Elisabet D. Membrane-like structure in the urinary bladder neck of a young cat: diagnosis and treatment usin balloon dilatation and a balloonexpandable metallic stent. J Feline Med Surg. 2017;3:232-5.

44 Leal LM, Crivelenti LZ, Cipolli VMM, Lima TB, Morato GO, Moraes PC. Uretrostomia pré-púbica após ruptura uretral em felino com doença do trato urinário inferior. Clin Vet. 2012;97:100-4.

45 Balakrishnan A, Drobatz KJ. Management of urinary tract emergencies in small animals. Vet Clin North Am Small Anim Pract. 2013;43:843-67.

46 Seitz MA, Creedon JMB, Drobatz KJ. Evaluation for association between indwelling urethral catheter placement and risk of recurrent urethral obstruction in cats. J Am Vet Med Assoc. 2018;252:1509-20.

47 Bartges JW, Kirk C, Lane IF. Update: management of calcium oxalate uroliths in dogs and cats. Vet Clin North Am Small Anim Pract. 2004;34:969-87.

48 Williams J. Surgical management of blocked cats: which approach and when? J Feline Med Surg. 2009;11:14-22.

49 Nye AK, Luther JK. Feline perineal urethrostomy: a review of past and present literature. Top Companion Anim Med. 2018;33:77-82.

50 Acar SA, Sarouglu M, Sadalak DJ. Prepucial urethrostomy performed using the coating technique (in Turkish). Turk J Vet Anim Sci. 2010;34:7-16.

51 Papazoglou LG, Basdani E. Perineal urethrostomy in the cat. Technique and complications. J Hellenic Vet Med Soc. 2011;62:150-60.

52 Bass M, Howard J, Gerber B, Messmer M. Retrospective study of indications for and outcome of perineal urethrostomy in cats. J Small Anim Pract. 2005;46:227-31.

53 Sousa-Filho RP, Nunes-Pinheiro DCS, Sampaio KO, Silva BCE, Cavalcanti GASA, Cunha MGMC. Clinical outcomes of 28 cats 12-24 months afer urethrostomy. J Feline Med Surg. 2019;23:1-8.

54 Paulo NM, Silva FF, Brito GA, Damasceno AD, Brito LAB, Freitas JS, et al. Reconstrução uretral com retalho autógeno de mucosa bucal após uretrostomia em cães. Acta Cir Bras. 2004;19:110-4. gatos. Arq Cienc Vet Zool UNIPAR. 2011;14:73-6. 
157 Lotan Y, Jiménez IB, Lenoir-Wijnkoop IL, Daudon M, Molinier L, Tack I, et al. Increased 2 water intake as a prevention strategy for recurrent urolithiasis: major impact of compliance on 3 cost-effectiveness. J Urol. 2013;189:935-9.

458 Forrester SD, Towell TL. Feline idiopathic cystitis. Vet Clin North Am Small Anim Pract. $5 \quad 2015 ; 45: 783-806$.

659 Sturgess K. Dietary management of canine urolithiasis. In Pract. 2009;31:306-12.

760 Ruda L, Heiene R. Short- and long-term outcome after perineal urethrostomy in 86 cats 8 with feline lower urinary tract disease. J Small Anim Pract. 2012;53:693-8. 